EOMmun: Communication et organisation

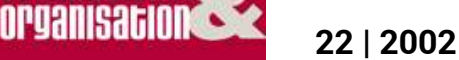

Interculturel et communication dans les organisations

\title{
La certification ISO 9000 en chine
}

Lihua Zheng

\section{OpenEdition}

Journals

Édition électronique

URL : http://journals.openedition.org/communicationorganisation/2782

DOI : 10.4000/communicationorganisation.2782

ISSN : 1775-3546

Éditeur

Presses universitaires de Bordeaux

Édition imprimée

Date de publication : 1 novembre 2002

ISSN : 1168-5549

Référence électronique

Lihua Zheng, "La certification ISO 9000 en chine », Communication et organisation [En ligne], 22 | 2002, mis en ligne le 27 mars 2012, consulté le 02 mai 2019. URL : http://journals.openedition.org/ communicationorganisation/2782 ; DOI : 10.4000/communicationorganisation.2782

Ce document a été généré automatiquement le 2 mai 2019

(c) Presses universitaires de Bordeaux 


\title{
La certification ISO 9000 en chine
}

\author{
Lihua Zheng
}

1 Depuis une dizaine d'années, avec la vogue de la mondialisation, de plus en plus d'entreprises chinoises sont passées aux normes ISO 9000, système international visant à établir une sorte de "management total de la qualité » (Beyer, 1999: 174). On peut discerner trois motivations principales qui poussent les entreprises chinoises à cette certification : aspiration à la modernisation de l'entreprise, contrainte imposée par les clients ou les partenaires qui exigent la possession du label comme une des conditions préalables pour l'établissement de la relation commerciale et recherche d'une image sociale octroyée par le label. Mais quelles sont les réalités après la certification? Est-ce que les normes ISO 9000 ont produit leurs effets dans ces entreprises? Quels sont les obstacles qu'elles ont rencontrés sur le terrain chinois et quelles sont les difficultés que les Chinois ont eues dans l'application de ce système? C'est à ces questions que nous allons essayer de répondre dans cet article. Nos analyses et nos réflexions s'appuient sur une enquête que nous avons effectuée en 2001 auprès des entreprises chinoises ayant obtenu la certification.

\section{Trois situations observées}

Dans toutes les entreprises chinoises ayant passé la certification ISO 9000, on ne trouve pas les mêmes effets de changements ni les mêmes efforts pour adopter le système. Selon notre enquête, nous pouvons relever trois situations qui correspondent à trois niveaux d'application des normes ISO 9000.

\section{Une réelle application des normes ISO 9000}

Il s'agit d'entreprises qui ont adopté les normes ISO 9000 et essaient de les appliquer à la lettre. C'est souvent le cas des entreprises étrangères implantées en Chine, des entreprises à capitaux mixtes ou des entreprises chinoises de grande envergure. Dans ces entreprises, on peut noter des changements importants apportés par la certification. 


\section{Des responsabilités réparties}

4 Dans cette première catégorie d'entreprises, on trouve une clarification des responsabilités exactes de chacun et une délimitation des frontières entre activités. Les documents de la certification ISO 9000 distribuent les responsabilités à chaque personne et à chaque département, de sorte que même la direction n'a pas le droit de prendre la décision à la place des autres. En cadrant et garantissant le droit de chaque personne et de chaque département, les règles réduisent celui de la direction. Les départements ont la responsabilité de toutes les décisions qu'ils prennent. Dans ce contexte ils ne veulent pas que le patron se mêle de leurs affaires parce qu'en cas de problème, le patron peut décliner sa responsabilité en disant: « d'après les documents, ce n'était pas mon travail. Vous avez eu tort de m'écouter ». Chaque département doit protéger son propre intérêt pour ne pas prendre en charge les problèmes causés par d'autres?

\section{Une logique professionnelle}

5 En réglant la relation entre les différentes parties de l'entreprise, les règles changent aussi cette dernière. Avant l'application des normes ISO 9000, l'entreprise était plutôt comme une grande famille pour qui le personnel avait de l'affection. C'est une logique des sentiments qui, comme son nom l'indique, est "profondément enracinée dans les sentiments et l'affectivité » et qui « représente les valeurs inhérentes aux relations entre hommes des différents groupes de l'entreprise » (Roethlisberger et Dickson. 1939). Mais avec les normes ISO 9000, ce sentiment de famille s'estompe petit à petit. Il est remplacé par une autre logique, celle du coût (une logique économique qui mesure en termes de prix de revient et plus généralement de résultats sur le marché ce qu'il est bon et mauvais de faire) et celle de l'efficacité (logique « technique » qui mesure l'adéquation des moyens aux fins).

\section{Un auto contrôle à tous les niveaux}

6 On trouve dans ces entreprises une sensibilisation à la qualité. Les opérateurs s'autocontrôlent car ce sont eux-mêmes qui décrivent leur travail et qui le signent. Les départements s'auto-contrôlent parce qu'ils sont responsables de tout le travail fourni par leurs membres. L'entreprise effectue un auto contrôle externe par des tests d'évaluation des partenaires et un auto contrôle interne par l'existence d'un département chargé de l'application des documents. Ce département est indépendant de tous les autres. Sa tâche consiste à inspecter le travail de tous les départements et à faire respecter les documents écrits. Il fonctionne plutôt comme un tribunal qui a même le droit de critiquer les erreurs du patron. Ainsi le patron de l'entreprise n'est plus comme l'empereur qui pouvait tout faire comme par le passé.

\section{Un double fonctionnement}

7 Selon notre enquête, certaines entreprises qui ont passé la certification ISO 9000 n'appliquent pas entièrement les normes du système : elles introduisent une partie de ces normes mais continuent à faire fonctionner l'ancien mode de management. Il y a une sorte de panachage. Si les normes profitent à la direction, elle les applique, comme par exemple, les normes concernant le contrôle de la qualité, le contrôle sur le personnel, 
etc.; si les normes touchent les droits de la direction, elle préfère les oublier et n'hésite pas à les enfreindre. Ces entreprises prennent en fait ce qui leur semble utile dans les normes ISO 9000 pour renforcer leur management traditionnel.

Ainsi, dans les pratiques quotidiennes de l'entreprise, le patron continue à lancer des ordres comme autrefois : «Dans notre société, d'après les référentiels ISO 9000, le projet doit être décidé conjointement par les team leaders d'échelons différents. Mais souvent, on ne fait pas comme ça. Par exemple, la décision pour la cotation doit être faite par plusieurs personnes mais en fait, le patron prend souvent tout seul la décision sans en discuter avec les autres » (F. Chinoise, 40 ans, cadre).

\section{La recherche d'une bonne image sociale}

On peut citer un cas représentant la troisième situation: une entreprise a demandé la certification, a rédigé les documents requis par l'audit, a payé les frais, l'organisme de certification est passé et finalement, le label a été accordé. Tout le monde était content et on a mis la plaque d'acier portant le label à l'entrée de l'entreprise ou dans le bureau du directeur. Puis, on a mis les documents dans les tiroirs et l'entreprise fonctionne exactement comme autrefois. Rien n'a changé, sauf l'étiquette de l'entreprise et avec elle un fardeau supplémentaire: il faut préparer des documents écrits pour l'évaluation de l'audit, pour lui attester qu'on a fait tout selon les normes. Ce genre d'entreprises a demandé la certification uniquement pour s'approprier une bonne image sociale qui lui servira plus tard lors de la promotion de l'entreprise et de ses produits. Souvent, la certification, au lieu d'apporter une amélioration dans le management et dans la qualité, entraîne l'entreprise dans une situation difficile, car elle doit dépenser de l'argent et de l'énergie pour faire face à l'audit, un pur geste de cinéma. Ainsi, $\mathrm{M}$. Wang, membre d'un organisme de certification nous confie que la certification ISO 9000 a provoqué une conséquence tragique dans beaucoup d'entreprises chinoises parce qu'après l'obtention du label, elles ont fait faillite.

\section{Des interprétations}

10 Nous voyons, au travers de la description des situations observées dans l'enquête, que si certaines entreprises chinoises réussissent plus ou moins à introduire les normes ISO 9000 dans leur management, il existe des entreprises qui ne les appliquent pas entièrement et celles qui demandent la certification uniquement pour le label. Cela veut dire qu'il y a des écarts entre la réalité de l'entreprise et les référentiels. En fait, ces écarts ne sont pas directement liés à ISO 9000, car avant même l'apparition des normes ISO, de nombreuses études ont montré les énormes écarts qui existent entre le travail « prescrit » et le travail réel exécuté par le salarié (Lasfargue, in Beyer. 1999 : 191). Ce phénomène d'écarts existe également en France (Cochoy et al.. 1998). ce qui varie entre les pays et entre les entreprises, ce sont des différences de degré. Certains auteurs pensent même que la règle, c'est la transgression des règles : «L'accomplissement effectif de l'activité ne s'accommode jamais d'un respect absolu des règles » (Girin et Grosjean, $1996: 5$ ).

11 La toute première raison de ce décalage entre les normes et la réalité est naturellement l'absence de volonté réelle dans l'introduction des normes, ce dont témoigne la troisième catégorie d'entreprises chinoises qui demandent la certification uniquement pour l'obtention d'une bonne image sociale générée par le label. Ainsi, elles ont mis en œuvre 
différents procédés qu'on peut qualifier de tricherie. L'un d'entre eux consiste, au lieu d'écrire ce qu'on fait dans la réalité, à écrire ce que l'audit demande. Un deuxième procédé consiste à demander aux opérateurs de fabriquer des documents écrits non pas d'après ce qu'ils font réellement mais d'après ce qu'ils doivent faire idéalement. Une autre technique consiste à désigner deux ou trois employés qui savent bien écrire au sein de chaque département et leur demander de rédiger à la place des autres. Enfin, un autre procédé qui va à l'extrême consiste à faire faire, c'est-à-dire à sous-traiter le travail de certification par une agence.

Dans notre enquête, nous avons découvert que la production de faux documents ne se limite pas aux entreprises qui passent la certification uniquement pour l'obtention du label mais qu'il s'agit plutôt d'un phénomène général, avec des variations quant à la gravité de la mystification. Ceci pourrait faire croire à la malhonnêteté des Chinois dans la certification ISO 9000. Or, ce serait, d'après nous, une approche simpliste que d'attribuer ce phénomène de tricherie entièrement aux défauts des Chinois. Derrière l'usage de ces procédés se cachent des éléments socioculturels qui constituent selon nous des raisons plus profondes des écarts entre la réalité et les normes ISO 9000.

\section{Des illusions des normes ISO 9000}

13 Nous allons d'abord chercher des causes du côté des normes ISO 9000 elles-mêmes recèlent des illusions rendant difficile leur application par rapport à la réalité. Les normes ISO 9000 reposent sur une conception instrumentale du langage. Elles s'appuient principalement sur l'écriture et considèrent les documents écrits comme des outils à l'égal des machines, le slogan étant «Ecrivez ce que vous faites et faites ce que vous écrivez »: "Les normes ISO 9000 véhiculent la croyance d'une possibilité de maîtrise du travail par le langage, dont l'idéal final est qu'un jour les entreprises réelles fonctionnent comme les procédures le disent » (Beyer, 1999 : 201). Le langage a, il est vrai, une force créatrice consistant à transformer les réalités du monde. Par exemple, une fois qu'il est écrit, chaque geste prend le sens d'être " conforme à ce que le service qualité dit » ou "non conforme", situation qui n'existait pas avant l'écriture. Mais le langage n'est pas tout à fait comme une machine et, contrairement à la croyance biblique selon laquelle «le verbe devient chair », le langage n'est pas réalité. Sa force créatrice ne réside pas dans sa forme mais dans les rapports sociaux qu'il cristallise.

Une partie des causes des écarts entre les normes ISO 9000 et la réalité sont directement liées aux caractéristiques inhérentes au langage sur lequel s'appuie le système. D'abord, ce slogan «Ecrivez ce que vous faites et faites ce que vous écrivez » fait croire qu'on peut tout écrire et que l'écrit peut correspondre point par point au faire et vice versa. Or le faire (l'action effective) et l'écrit (le texte de la règle) ne sont pas les mêmes : l'action est toujours située, c'est-à-dire d'une certaine manière, unique, alors que le texte est censé couvrir la généralité des cas. Le sens que prendra une phrase ne peut être défini à l'avance dans l'abstrait, mais prendra son sens dans la situation concrète où elle sera prononcée ou dans laquelle elle figurera par écrit. On ne saurait donc trop s'étonner de la dérive des normes car le respect à la lettre de celles-ci risque d'entraver la débrouillardise quotidienne impliquée par tout travail concret, et les compromis qu'il est souvent nécessaire de négocier entre plusieurs contraintes souvent opposées. Différents auteurs soulignent ainsi les risques de paralysie et signalent que la meilleure façon d'arrêter la production serait de faire la "grève du zèle des normes ", en les appliquant à la lettre 
(Lebaube, in Beyer. 1999 : 190). Ensuite, il serait illusoire de croire qu'on peut mettre par écrit tout ce qu'on fait. Une partie des gestes, incorporée et devenue automatique, est basée en fait sur un savoir-faire acquis et transmis par expérience, non formalisée, qu'on ne connaît soi-même qu'en partie et que l'on sent sans pouvoir forcément l'expliciter. Prenons l'exemple de la pratique de l'acupuncture. Les étudiants européens qui étudient la médecine chinoise en Chine demandent souvent à leur professeur chinois de préciser la profondeur exacte en millimètres à laquelle ils doivent enfoncer l'aiguille. Or, la précision détruit la pertinence de la technique, car la profondeur de l'aiguille dépend de la combinaison d'un certain nombre de facteurs comme la nature de la maladie, le climat, l'âge du patient, son sexe, son état de santé, etc. Enfin, les normes ISO 9000 étant basées sur l'écriture, elles reposent avant tout sur le sens des mots qui prêtent nécessairement à des interprétations différentes, notamment lorsqu'on passe d'une langue à une autre. Par exemple, les Chinois et les Français peuvent diverger dans leurs manières d'interpréter des concepts de base comme entreprise, qualité, opérateur, contrôle, etc. Même à l'intérieur d'une culture, il peut y avoir des confusions dans l'interprétation des termes utilisés dans les cahiers des charges, les contrats et les appels d'offres.

Une autre illusion fondamentale des normes ISO 9000, c'est de croire que devant les documents écrits considérés comme faisant loi, tout le monde est égal, du PDG aux ouvriers, puisque tous doivent écrire et se conformer à l'écriture. Or, dans les entreprises, les employeurs et les employés ne se trouvent jamais sur le même pied d'égalité, les premiers cherchant toujours à maximiser les efforts des seconds et ces derniers ayant toujours l'impression d'être exploités par les premiers. Les normes ISO 9000 incarnent en fait les rapports de force entre ceux qui prescrivent les règles et ceux qui doivent les subir. Au cours de notre enquête, nous avons pu relever des attitudes différentes des Chinois à l'égard des normes ISO 9000. D'une part, certains dirigeants et cadres se montrent enthousiasmés par la certification, disant qu'elle est nécessaire et exigée par la modernisation ou la survie de l'entreprise. D'autre part, les ouvriers trouvent la certification inutile, contraignante, voire même embêtante. Si la direction choisit de faire certifier l'entreprise, c'est en partie parce qu'elle voit se réaliser son rêve de voir qu'un jour, ses employés travailleront de manière entièrement conforme à ses directives consignées dans les «manuels qualité ». Les cadres, eux, voient dans la certification un moyen de mieux contrôler le travail de ceux qu'ils encadrent, voire un instrument permettant une certaine reprise en main. Par la certification, la partie dominante de l'entreprise impose en fait à la partie dominée une nouvelle morale du travail, un ensemble de normes de conduite et de comportements à respecter, des directives destinées à planifier et donc à contrôler les gestes quotidiens. Les ouvriers, de leur côté, ne se laissent pas faire. Ils acceptent d'écrire certaines manières de faire en cherchant dans l'écriture des avantages comme l'amélioration des conditions de travail et la reconnaissance de leur compétence et de leur légitimité, mais ils refusent de tout écrire car exposer tout, c'est perdre un peu de sa marge de manœuvre et de son pouvoir sur le poste de travail, c'est aussi donner prise à un contrôle ultérieur sur le rapport entre activité déclarée et activité effective et livrer un bagage de connaissances qui pourrait servir à former un éventuel remplaçant. Ainsi, on peut constater dans les entreprises certifiées des choses qui sont faites mais qui ne sont pas notifiées et donc qui sont difficilement réalisables par les personnes qui viennent en remplacement. On remarque également une non conformité générale aux fiches d'opération. Ceci peut être expliqué, du point de vue du rapport social entre ceux qui édictent les règles et ceux qui sont 
censés devoir les appliquer, par le fait que ne pas respecter les règles à la lettre, c'est ne pas se soumettre entièrement aux autres.

\section{Des obstacles culturels}

16 écrits. L'écriture constitue le fondement du bon fonctionnement du système. Or, l'acte d'écrire, comme tout acte social, est soumis à des contraintes socioculturelles qui font que n'importe qui ne peut écrire n'importe quoi à n'importe qui dans n'importe quelle situation. Dans la relation entre le langage et la société, la dernière détermine le premier et se pose normalement comme une condition préalable : un directeur parle comme un directeur parce qu'il est directeur. Sa façon de parler est déterminée par son statut social, les présupposés socioculturels qu'il se fait de son statut, les habitudes linguistiques du groupe auquel il appartient, etc. Et tout cela existe avant son acte de parole. De même, l'écriture introduite par les normes ISO 9000 n'est pas basée sur le néant. Elle est d'abord une création des Occidentaux : «Les normes ISO 9000 sont mises au point par l'ISO, créée en 1947, dans laquelle sont représentés les principaux organismes de normalisation de nombreux pays au sein desquels les États-Unis ont un rôle hégémonique » (Cruchant, in Beyer, 1999: 175). Elle est ensuite liée à une culture d'entreprise marquée du seau occidental : «Les normes ISO produisent donc des effets organisationnels institutionnels et réglementaires, et comportent des éléments d'une nouvelle culture d'entreprise qui se réfère à l'Europe » (Beyer, 1999: 195). On peut lire derrière cette nouvelle écriture la prégnance «d'un certain esprit protestant, dont la rigueur et l'austérité de ces normes témoignent » (ibid: 203). On peut dire que cette écriture a comme arrière-plan culturel l'esprit analytique des Occidentaux, leur préférence pour la déduction, leur tendance à la rationalité, leur tradition juridique insistant sur des contrats qui impliquent la définition claire des droits et des obligations de différentes parties, la précision des responsabilités des uns et des autres, les traces, les signatures, un contrôle basé sur des textes, les effets irréversibles, etc.

Regardons maintenant la situation en Chine. Les entreprises chinoises sont en quête du label ISO 9000 pour différentes raisons que nous avons mentionnées au début de l'article. Elles ne sont pas suffisamment conscientes du fait qu'en réalité, les conditions socioculturelles qui supportent ce système n'existent pas en Chine. ISO 9000 en Chine est un peu comme un arbre replanté ne retrouvant pas le terreau nécessaire pour sa survie. Nous voulons dire par là que les normes ISO 9000. produit de la culture occidentale, ne manquent pas de se heurter en Chine à des obstacles culturels. une logique de moralité et de sentiments qui gère les relations interpersonnelles à travers une conscience interne de l'homme contrairement à la logique de la loi qui les gère à travers une obligation externe (Zhang et Fang, 1989: 8). Pour mieux montrer cela, il nous semble nécessaire de passer en revue quelques préceptes du confucianisme, courant de pensée qui a profondément marqué la mentalité des Chinois.

19 Pour l'organisation de la vie sociale, le confucianisme prône l'harmonie et la bonne entente dans les cinq rapports fondamentaux qu'engage un homme : entre le prince et le ministre, entre le père et le fils, entre le mari et la femme, entre l'aîné et le cadet, et entre amis. Chaque personne a un «statut » associé à la personne avec qui elle forme une « un couple » (par exemple, l'un est père, l'autre fils). Ce statut indique la place qu'un individu 
occupe dans la société et le type de rapport qu'il doit entretenir avec les autres et fixe à chacun ses droits et obligations : le père doit montrer de la bienveillance et le fils de la piété filiale: l'âné, de la gentillesse et le cadet du respect; le mari, le sens des responsabilités et la femme l'obéissance; le vieux, l'amabilité et le jeune la docilité ; le prince, de l'indulgence et le ministre de la fidélité, telles sont les dix homologies de valeurs à respecter. Nous voyons par là qu'il s'agit d'une morale de type familial. Le rapport entre le père et le fils en constitue la base. Parmi ces cinq rapports, trois s'appliquent à la famille (entre le père et le fils, le mari et la femme, l'aîné et le cadet). Les deux autres (entre le prince et le ministre, et entre amis) sont en réalité des rapports familiaux au sens élargi. En effet, le système familial constitue le fondement de la société chinoise : « Le système familial et le système villageois, tel un système familial élargi » dit Lin. chercheur chinois. "peuvent expliquer tous les phénomènes de la vie sociale chinoise" (1990: 161). Comme la gestion entre les membres d'une famille est principalement régie par la moralité et les sentiments, l'organisation de la société qui est en fait une famille élargie, est basée sur une logique de sentiments qui implique, contrairement à la gestion par contrats, autorité personnelle, confiance, liens de sang et de réseau, bonne entente et harmonie dans les relations, oralité, implicite, etc. On peut relever au moins trois obstacles que la culture chinoise oppose à l'application des normes ISO 9000. D'abord, la gestion des relations sociales chinoises, notamment entre les proches comme les membres de la famille, collègues, etc. repose principalement sur la parole caractérisée par l'implicite, l'imprécis et l'environnement situationnel. Or. les normes ISO 9000 demandent de tout écrire, de tout expliciter et de tout tracer en dehors de toute situation. C'est pour cette raison que certains Chinois trouvent les normes ISO 9000 « froides » et « sans sens de l'humain ». Ensuite, dans la vie sociale chinoise, les Chinois insistent sur les réseaux de relations alors que les normes ISO 9000 laissent justement ces réseaux dans l'ombre et font comme s'ils n'existaient pas. Enfin, il y a le "sens de l'autorité personnelle». Les normes ISO 9000 font croire que ce sont les documents qui contrôlent le travail de l'entreprise. Les Chinois, eux, pensent que celui qui fait vraiment force de loi, c'est le patron considéré comme ayant le même statut que le père de famille et ils ne pensent pas que l'autorité de ce dernier peut être facilement remplacée par les normes ISO 9000. Au cours de notre enquête, nous avons constaté que souvent les opérateurs ne suivent pas les règlements écrits mais n'osent pas contrarier les ordres de la direction. Une raison est qu'ils comprennent bien la situation dans l'entreprise : les règles sont faites par l'homme et appliquées par l'homme et l'homme le plus puissant est le patron ; les règles sont « mortes » alors que l'homme est « vivant »; si l'on veut continuer à rester dans l'entreprise, il faut suivre l'homme et non pas les règles. "On dit souvent que les normes ont une autorité absolue mais en fait dans une entreprise, celui qui a vraiment de l'autorité, c'est le directeur. Les mesures de punition de la normalisation ne suffisent pas. Par exemple, quand nous trouvons dans le travail des problèmes émanant d'une section, nous lui passons, selon les normes, une note pour qu'elle corrige des fautes, mais il arrive souvent qu 'elle ne nous réponde pas. Mais dès que le directeur intervient, les problèmes se résolvent rapidement. Dans ce cas-là, la normalisation n'est pas aussi autoritaire que la parole du directeur. Qui ose enfreindre l'ordre de la direction? Elle a toujours le droit de vous renvoyer. » (F, Chinoise, 25 ans, employée). En effet, face à deux ordres contradictoires, l'un défini par les normes ISO 9000, l'autre dicté par le directeur, les opérateurs chinois choisissent de suivre les ordres du dernier aux dépens des premières. 


\section{Des conditions économiques insuffisantes}

Incompatibilité des structures d'entreprise

20 structure propre aux entreprises de type européen, ayant une séparation stricte entre leurs domaines d'activité respectifs et connaissant une forte professionnalisation de leurs activités d'organisation et d'encadrement (Beyer, 1999: 179). Or. cette infrastructure n'est pas tout à fait compatible avec celle d'une entreprise chinoise. Ouand on introduit le système, on ne se rend pas compte qu'une entreprise chinoise et une entreprise européenne peuvent différer par leurs organigrammes, la structure des échelons, la répartition des postes, etc. Ce problème d'incompatibilité est le plus observé dans les joint-ventures qui se trouvent être des filiales des entreprises européennes : la société mère impose des documents écrits à toutes ses filiales car elle veut que l'entreprise soit certifiée de la même manière partout dans le monde : «Notre société a copié des documents de la société mère française. Mais la structure de l'entreprise n'est pas la même que chez nous. Par exemple, selon les documents écrits, plusieurs team leaders interviennent dans le lancement $d$ un nouveau produit. I un s'occupe de l'exploitation du produit, un autre de la fixation du prix et un autre encore de la mise en production. Chacun, à la fin de son travail, doit signer un papier. Mais ici, je suis seule à m'occuper de toutes les étapes. Comment voulez-vous que j'applique ci la lettre des documents? D'après ce que je sais. $80 \%$ des entreprises fabriquent des documents pour la visite de l'audit. La question est de savoir combien elles en fabriquent " (F, Chinoise, 38 ans. chef de projets)

\section{Manque du feed-back du marché}

21 Dans le système ISO 9000, le feedback des clients et du marché est important. Avant ISO 9000, les entreprises et le marché étaient presque comme deux vêtements qui ne s'harmonisaient pas toujours. Les normes ISO 9000 permettent un meilleur ajustent parce qu'un des objectifs principaux des procédures ISO 9000 est de donner confiance et satisfaction aux clients. Leurs remarques servent de référence pour l'amélioration de la qualité de l'entreprise et elles peuvent guider la direction dans le développement de l'entreprise. Dans le système ISO 9000 au sens complet, le feed-back, c'est-à-dire les conseils des clients, doit participer à tous les processus de la production. D'après le feedback, les entreprises peuvent juger si l'orientation de leur production correspond au marché, et s'il y a des problèmes, dans quel sens elles doivent s'orienter. Dans un esprit de coopération, l'entreprise a besoin de recueillir les remarques écrites des clients. Ainsi, dans la situation économique du marché, la production d'une marchandise est loin d'être l'affaire d'une seule entreprise. Les consommateurs et les entreprises deviennent deux parties qui s'entraident pour atteindre leur propre but. Le processus de conception et d'appropriation d'un nouveau produit n'est pas linéaire mais constitue un double boucle rétroactive. Mais d'après l'enquête que nous avons effectuée, ce feedback manque à la plupart des entreprises passant la certification ISO 9000 parce que les clients n'aiment pas écrire ou ne veulent pas coopérer. "Quelquefois, les clients de notre entreprise trouvent des problèmes dans nos produits, ils en sont mécontents, mais ils ne les notent pas dans les formulaires prévus à cet effet ou ils mentionnent seulement, en ce qui concerne la qualité, "bonne» ou "mauvaise ". Cela nous empêche de connaître clairement les problèmes et d'y apporter des solutions». Comme le feed-back du marché devient de plus en plus indispensable pour 
l'entreprise, cette dernière fait parfois appel à une agence qui s'occupe particulièrement de l'enquête sur les opinions des clients et des consommateurs. Mais les informations fournies sont souvent peu fiables: "Nous avons demandé à des organismes de mener une enquête pour notre société afin de savoir si les consommateurs sont satisfaits ou non de nos produits et pour avoir connaissance d'éventuels problèmes. Mais chaque fois les résultats sont faux et cela peut poser de gros problèmes pour l'amélioration permanente de la qualité de nos produits " (M, Chinois. 40 ans, cadre).

Cela nous montre que l'écriture dans le système ISO 9000 n'est pas seulement l'affaire de l'entreprise. Elle est l'affaire de toutes les personnes et de toutes les parties qui sont en relations directes ou indirectes avec l'entreprise. Ici, le sens de l'écriture devient beaucoup plus large. L'entreprise, pour appliquer les normes ISO 9000, a besoin non seulement de la coopération de son personnel, mais aussi de celle de ses partenaires et ses clients. Cela nous conduit à une autre remarque plus importante: Les normes ISO 9000, pour pouvoir fonctionner, ont besoin d'un environnement socio-économique qui les soutienne: la constitution de cet environnement requiert un certain degré de développement économique, une certaine maturité du marché et une prise de conscience de l'ensemble de la population.

\section{Conclusion}

Nous avons montré dans cet article, à travers la description des réalités de la certification ISO 9000 en Chine, qu'il existe de grands écarts entre les normes prescrites et leur application dans les entreprises chinoises. Nous avons essayé d'interpréter ces écarts sous différents angles. D'après nous, interviennent dans ce phénomène d'écarts non seulement les représentations que les Chinois se font des normes ISO 9000, les objectifs qu'ils se fixent pour la certification, les procédés mis en pratique, mais aussi et surtout l'environnement socio-économico-culturel qui constitue l'arrière-plan du système. Les entreprises chinoises se trouvent en fait devant un dilemme difficile à résoudre. Pour s'intégrer au marché international et à la mondialisation économique, elles se voient dans l'obligation urgente de demander la certification ISO 9000, mais ce système, lui-même produit de la culture européenne et ayant pour support des structures entrepreunariales de type européen, est loin de correspondre à la mentalité des Chinois, ni aux pratiques traditionnelles des entreprises chinoises, ni à l'ambiance socio-économique du pays. Cela ne veut pas dire qu'à cause de l'incompatibilité des normes ISO 9000 avec la culture chinoise et les conditions économiques encore insuffisantes du pays, la Chine ne devrait pas introduire le système. Bien au contraire, nous devons redoubler d'efforts dans l'adoption des normes internationales d'abord parce que face à la mondialisation, nous n'avons pas d'autre choix, puis parce que le système ISO 9000, saisi dans son rôle de médium, possède une force créatrice, c'est-à-dire qu'il peut influencer des mentalités, modifier des comportements et changer des pratiques d'entreprise tout comme l'apparition de l'écriture, du papier et d'internet ont apporté de profonds changements au monde. 


\section{BIBLIOGRAPHIE}

BEYER, F. M., 1999, Au-delà de la qualité : démarches qualité, conditions de travail et politiques du bonheur. Paris : Syros.

COCHOY. F., GAREL J-P., TERSSAC G., 1998, Comment l'écrit travaille l'organisation : le cas des normes ISO 9000. In Revue française de sociologie, vol. XXXIX. $\mathrm{n}^{\circ} 4$.

GIRIN. J.. et GROSJEAN, M. (éd.), 1996, La transgression des règles au travail, Paris : Éditions CNRS.

LIN, Y.T., 1990. Mon pays et mon people. Beijing : Edition du théâtre de Chine.

ROETHLISBERGER. F-J., et DIKSON. V., 1939, Management and the worker, Cambridge: Harvard University Press.

ZHANG. D-N. et FANG L-T., 1989, Pensées philosophiques de la Chine antique. L'intelligence du people chinois. Shanghai : Éditions du people de Shanghai.

\section{RÉSUMÉS}

Avec la vogue de la mondialisation, de plus en plus d'entreprises chinoises sont passées aux normes ISO 9000. Une enquête menée par l'auteur et son équipe a montré qu'il existait des écarts entre la réalité de l'entreprise et les référentiels. Selon l'auteur, ces écarts peuvent s'expliquer d'une part par les principes de base des normes elles-mêmes qui recèlent des illusions et d'autre part, par des différences socioculturelles.

As mondialization is in vogue, more en more Chinese firms conform to ISO 9000 norms. A survey led by the author and his research team showed that differences exist between the definition of norms and the firms'realities. According to the author, theses differences can be explained, on the one hand by the basic characteristics of the ISO 9000 norms that contain illusions and on the other hand by sociocultural differences.

\section{INDEX}

Mots-clés : ISO 9000, communication écrite, culture. Chine

\section{AUTEUR}

\section{LIHUA ZHENG}

ZHENG Lihua est professeur de sociolinguistique à l'Université des Etudes Etrangères du Guangdong, directeur du CERSI (Centre de Recherche sur l'Interculturel) et chercheur au Center for Linguistics and Applied Linguistics de cette même université. Il est l'auteur de Les Chinois de Paris et leurs jeux de face (L'Harmattan. 1995), de Langage et interactions sociales (L'Harmattan. 1998). de Chine-France, approches interculturelles en économie, 
littérature, pédagogie, philosophie et sciences sociales (L'Harmattan, 2000) et de Entreprises et vie quotidienne en Chine (L'Harmattan. 2002). 